\title{
Pilonidal Sinus: Karydakis Technique is as yet an Undemanding Compelling Alternative in Current Practice
}

ISSN: 2578-0379

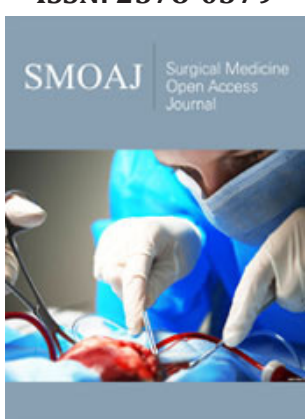

*Corresponding author: Elmutaz Kanani, General Surgery Department, Khoula Hospital, Oman.

Submission: 非 August 28, 2020

Published: 温 September 04, 2020

Volume 3 - Issue 4

How to cite this article: Elmutaz Kanani, Ahad Ahmed. Pilonidal Sinus: Karydakis Technique is as yet an Undemanding Compelling Alternative in Current Practice. Surg Med Open Acc J. 3(4). SMOAJ. 000569. 2020. DOI: 10.31031/SMOAJ.2020.03.000569

Copyright@ Elmutaz Kanani, This article is distributed under the terms of the Creative Commons Attribution 4.0 International License, which permits unrestricted use and redistribution provided that the original author and source are credited.

\author{
Elmutaz Kanani* and Ahad Ahmed \\ General Surgery Department, Oman
}

\begin{abstract}
Background: Pilonidal sinus disease is a soft tissue infection. It presents either acutely with abscess or the chronic form of sinus formation. The disease affects multiple body regions but the commonest is the sacrococcygeal region. There are different treatment strategies ranging from simple incision and drainage of an abscess to complex constructive procedures.
\end{abstract}

Objectives: The aim of this study is to share our experience and spotlight that the Karydakis procedure is as yet a legitimate and relatively simple effective treatment alternative for the non-specialized surgeon.

Materials and Methods: In this retrospective study the management of patients presenting with sacrococcygeal pilonidal sinus disease to Khoula hospital (Oman) over a period of 10 years is analyzed. Data, being retrieved from the electronic patient's records, covered the presentation, management, complications, and follow up.

Results: A total of 333 patients were included. The commonest presentation was of chronic sinus and the main procedure was the Karydakis operation (93.1\%). Postoperative complications developed in 24.6\% of all treated patients, and the recurrence rate was $4.5 \%$

Conclusion: The Karydakis operation is a relatively simple procedure to achieve cure in a reasonable time frame with acceptable risks of postoperative complications and recurrence.

Keywords: Advancement flap; Karydakis; Pilonidal; Recurrence

\section{Introduction}

Pilonidal disease is a commonly encountered disease with worldwide distribution. It is generally considered to be an acquired response to local infection seeping into the subcutaneous fatty tissue which contains hair follicles. It results in the formation of sinuses (tunnels) or cysts. Further it is classified into acute or chronic infection. Pilonidal disease affects different areas of the body, namely natural clefts or folds. It is predominantly seen in sacrococcygeal region hence it will be the focus of this study. In the literature the exact etiology of the disease is not fully understood, however over the years observational theories of pathogenesis had emerged. That improved the understanding of the disease etiology and contributed to the construction of the surgical management framework.

Initially Herbert Mayo, British Physiologist, Anatomist and Surgeon $\left(3^{\text {rd }}\right.$ April $1796-28^{\text {th }}$ June 1852), described it as (a sinus containing hair follicles located in the sacrococcygeal region in a woman) in 1833 [1]. In 1880 Hodges named the disease with the statement of (I venture to give the name of pilo-nidal (pilus: a hair, nidus: a nest) sinus to this rather singular lesion) $[1,2]$. It is a common disease, but its treatment is a challenging surgical task. Complications and treatment failure i.e. recurrence are not uncommon. The surgical interventions were designed based on clinical presentations and the disease etiology. Adherence to these surgical principles is the key to successful treatment [3]. Several pathogenesis hypotheses were suggested. Some theories staged hyperkeratosis as first step that results in dilatation and rupture of a hair follicle [4]. Contradicting and popular hypothesis proposed that the sinus formation is a sequela of skin breach by hair that have been shed or cut $[3,5]$. Ultimately, 
there are various presentations of the pathology. It may present as painful natal cleft abscess, commonly bulging lateral to midline. It may also present as a long standing natal cleft discharging sinus. A chronic sinus may be accompanied by laterally-located secondary openings or granuloma. Furthermore, asymptomatic form is another identified presentation characterized by incidental finding of one or more non-inflamed pits in the natal cleft.

Much has been written about pilonidal disease treatment globally featuring different surgical approaches affirming that it is a challenging task. Traditionally the mainstay management was based on excision of the sinus tracts followed by primary closure facilitating healing by primary intention or leaving the wound open to heal by secondary intention [6]. At present different treatment methods are available. They range from simple abscess drainage, to minimally invasive techniques, instillation of phenol, total excision with primary closure, flaps, to video-assisted and laser techniques. Each of these approaches has its advantages and disadvantages. The presence of this wide scope of alternative approaches can't be attributed to the incredible accomplishment of any of them, but instead the disappointment with the blemished consequences of every one of them [7-9]. In this study we are presenting our experience in the management of pilonidal sinus disease over the period from 2005 to 2015 . Our standard procedure is the Karydakis' procedure which is the basic off-midline procedure [5]. Our aim is to draw attention to an old; but still valid and successful treatment method and contribute to the development of international consensus about the optimal management of pilonidal sinus disease.

\section{Materials and Methods}

This is a retrospective observational study. Data was collected from hospital electronic records after obtaining the ethical approval.
All patients with sacrococcygeal pilonidal disease who were treated by the general surgery department in Khoula hospital (Muscat, Oman) from 2005 to 2015 as in-patients were retrospectively studied. Data were extracted from the electronic patient's records. After exclusion of incomplete patient's records, the data for 333 patients was analyzed. Demographics, presentation, the surgical procedure performed, the length of hospital stay, follow up and complications including recurrence were examined. The KruskalWallis test and Fisher Exact test were used for inter/bilateral group comparisons. The frequencies, minima, maxima, mean, and averages are reported for demographic data. $p<0.05$ was considered statistically significant.

\section{Operative Procedure}

Patients who presented with chronic disease (i.e. sinus) together with those who had an incision and drainage of abscess with recurrence were subjected to Karydakis operation. Patients were operated by all the members of the department at different grades, but the technique was standardized among the department members. All patients were operated under general anesthesia in prone position with the buttocks strapped apart. The procedure tasks performed were excision of sinuses down to pre-sacral fascia through an un-equal ellipse, advancement flap, and off-midline closure (Figure 1). A suction drain was always used and removed once its discharge is below 20-30 ml over 24 hours. The patients are discharged home the same day of drain removal and requested to remove the sutures on the $12^{\text {th }}-14^{\text {th }}$ post-operative day in their nearby health center. They are routinely discharged with analgesia only; however, in certain situations they were supplemented with an oral antibiotic. They were usually reviewed in the outpatient clinic 2-3 weeks post-operatively. Reappearance of sinuses with or without discharge at any point of time after post-operative complete healing of wound was considered as recurrence.

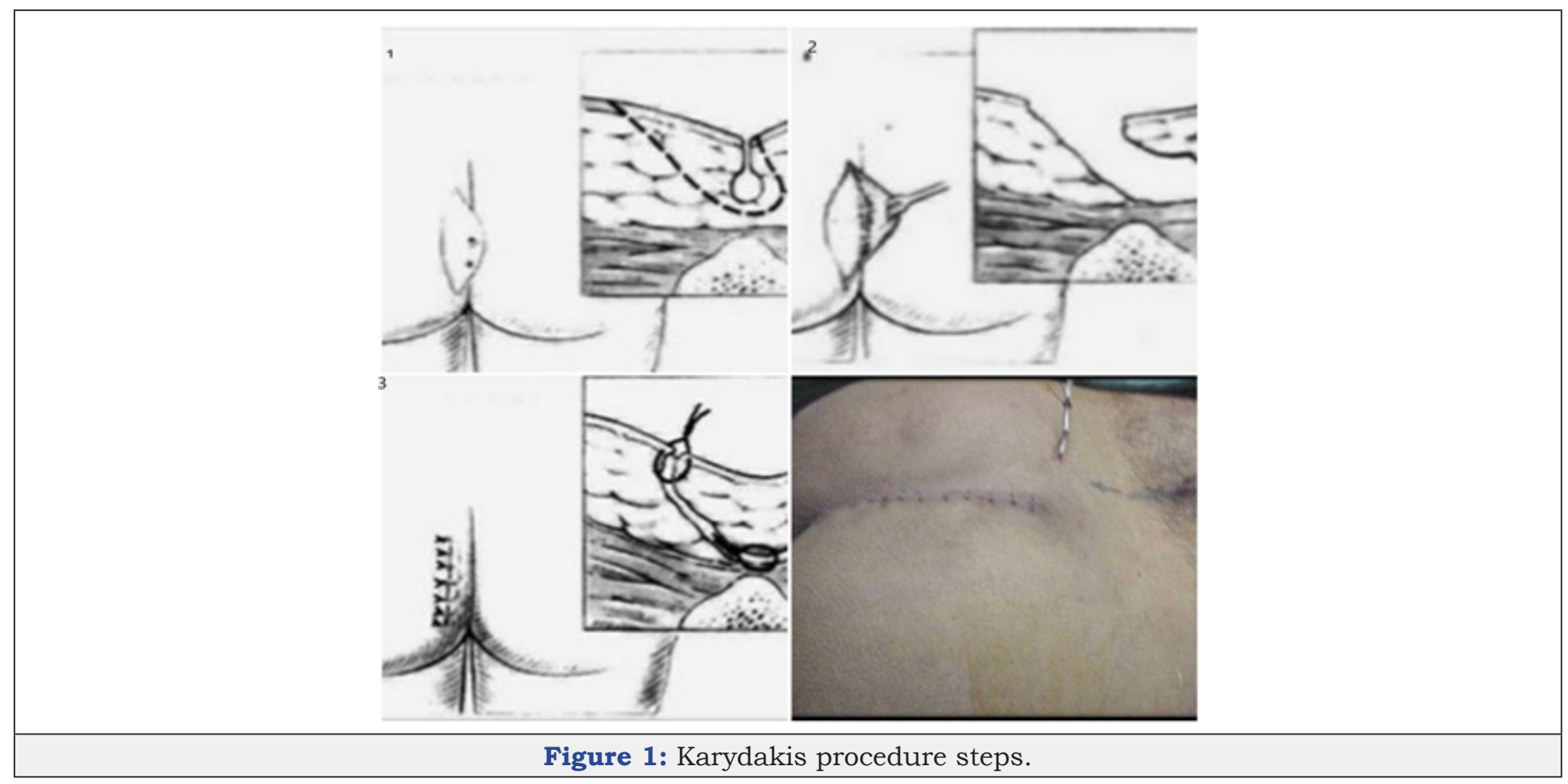




\section{Results}

A total number of 333 patients were included in the study. They presented with pilonidal sinus (259 patients: $77.8 \%$ ), pilonidal abscess (41 patients (12.3\%), and recurrence of the disease from a previous surgery (33 patients (9.9\%). The mean age at presentation was 24.3 years (13-34). Most patients (310 patients: 93.1\%) were subjected to Karydakis operation during the study period and the hospital stay for this group was 5.36 days (Table 1). Remaining patients were treated by excision and healing by secondary intention (10 patients; $3 \%$ ) while 8 patients $(2.4 \%$ ) had been managed by excision and Limberg flap reconstruction. Complications occurred in 82 patients $(24.6 \%)$; wound healing related complications were $10.5 \%$, and the recurrence rate was $4.5 \%$ (14 patients) (Table 2). Management of the complications is shown in Figure 2. The mean follow up time was 11.2 weeks (minimum 1 week, maximum 3.5 years).

Table 1: Baseline characteristics of the patients according to Presentation and Surgical Procedure.

\begin{tabular}{|c|c|c|c|c|c|}
\hline \multirow{2}{*}{ Characteristics } & \multicolumn{4}{|c|}{ Surgical Procedures } & \multirow{2}{*}{ p. Value } \\
\hline & Open & Karydakis & Limberg & Secondary Closure & \\
\hline Number of Patients & $10(3 \%)$ & $310(93.1 \%)$ & $8(2.4 \%)$ & $5(1.5 \%)$ & \\
\hline \multicolumn{5}{|c|}{ Age } & \multirow{5}{*}{$0.077 \#$} \\
\hline Mean & 27.9 & 23.74 & 24.75 & 20.8 & \\
\hline Median & 29 & 23 & 24.5 & 21 & \\
\hline Minimum & 19 & 13 & 15 & 18 & \\
\hline Maximum & 34 & 58 & 36 & 23 & \\
\hline \multicolumn{5}{|c|}{ Hospital Stay } & \multirow{5}{*}{$0.139 \#$} \\
\hline Mean & 8.7 & 5.36 & 7.25 & 8 & \\
\hline Median & 3.5 & 5 & 6.5 & 5 & \\
\hline Minimum & 3 & 1 & 4 & 4 & \\
\hline Maximum & 20 & 46 & 15 & 17 & \\
\hline \multicolumn{5}{|c|}{ Gender } & \multirow{3}{*}{$0.952^{*}$} \\
\hline Male & $6(2.9 \%)$ & $193(92.8 \%)$ & $6(2.9 \%)$ & $3(1.4 \%)$ & \\
\hline Female & $4(3.2 \%)$ & $117(93.6 \%)$ & $2(1.6 \%)$ & $2(1.6 \%)$ & \\
\hline \multicolumn{5}{|c|}{ Presentations } & \multirow{4}{*}{$0.014^{*}$} \\
\hline Abscess & $3(7.3 \%)$ & $37(90.2 \%)$ & $0(0.0 \%)$ & $1(2.4 \%)$ & \\
\hline Sinus & $7(2.7 \%)$ & $245(94.6 \%)$ & $5(1.9 \%)$ & $2(0.8 \%)$ & \\
\hline Recurrence & $0(0.0 \%)$ & $28(84.8 \%)$ & $3(9.1 \%)$ & $2(6.1 \%)$ & \\
\hline
\end{tabular}

\#Based on Kruskal Wallis Test

*Based on Fisher Exact Test

Table 2: Complications according to the initial presentation and the surgical procedure performed.

\begin{tabular}{|c|c|c|c|c|c|c|}
\hline \multirow{2}{*}{\multicolumn{2}{|c|}{ Characteristics }} & \multicolumn{4}{|c|}{ Procedures } & \multirow[b]{2}{*}{ p. value* } \\
\hline & & \multirow{2}{*}{$\begin{array}{c}\text { Open } \\
1 \\
\end{array}$} & \multirow{2}{*}{$\begin{array}{c}\text { Karydakis } \\
5 \\
\end{array}$} & \multirow{2}{*}{$\begin{array}{c}\text { Limberg } \\
0 \\
\end{array}$} & \multirow{2}{*}{$\begin{array}{c}\begin{array}{c}\text { Secondary } \\
\text { Closure }\end{array} \\
0 \\
\end{array}$} & \\
\hline \multirow{3}{*}{$\begin{array}{l}\text { Surgical site } \\
\text { Infection }\end{array}$} & Abscess & & & & & \multirow{3}{*}{0.301} \\
\hline & Sinus & 1 & 17 & 1 & 0 & \\
\hline & Recurrence & 0 & 2 & 1 & 0 & \\
\hline \multirow{3}{*}{ Dehiscence } & Abscess & 0 & 0 & 0 & 0 & \multirow{3}{*}{-} \\
\hline & Sinus & 0 & 6 & 0 & 0 & \\
\hline & Recurrence & 0 & 0 & 0 & 0 & \\
\hline \multirow{3}{*}{ Chronic Pain } & Abscess & 1 & 0 & 0 & 0 & \multirow{3}{*}{0.5} \\
\hline & Sinus & 0 & 2 & 0 & 0 & \\
\hline & Recurrence & 0 & 1 & 0 & 0 & \\
\hline \multirow{3}{*}{ Delayed Healing } & Abscess & 0 & 5 & 0 & 0 & \multirow{3}{*}{0.999} \\
\hline & Sinus & 0 & 20 & 1 & 1 & \\
\hline & Recurrence & 0 & 2 & 0 & 0 & \\
\hline
\end{tabular}




\begin{tabular}{|c|c|c|c|c|c|c|}
\hline \multirow{3}{*}{ New Recurrence } & Abscess & 0 & 1 & 0 & 1 & 0 \\
\cline { 2 - 7 } & Sinus & 0 & 11 & 1 & 0 \\
\cline { 2 - 7 } & Recurrence & 0 & 2 & 0 & 0.45 \\
\hline
\end{tabular}

*Based on Fisher Exact Test

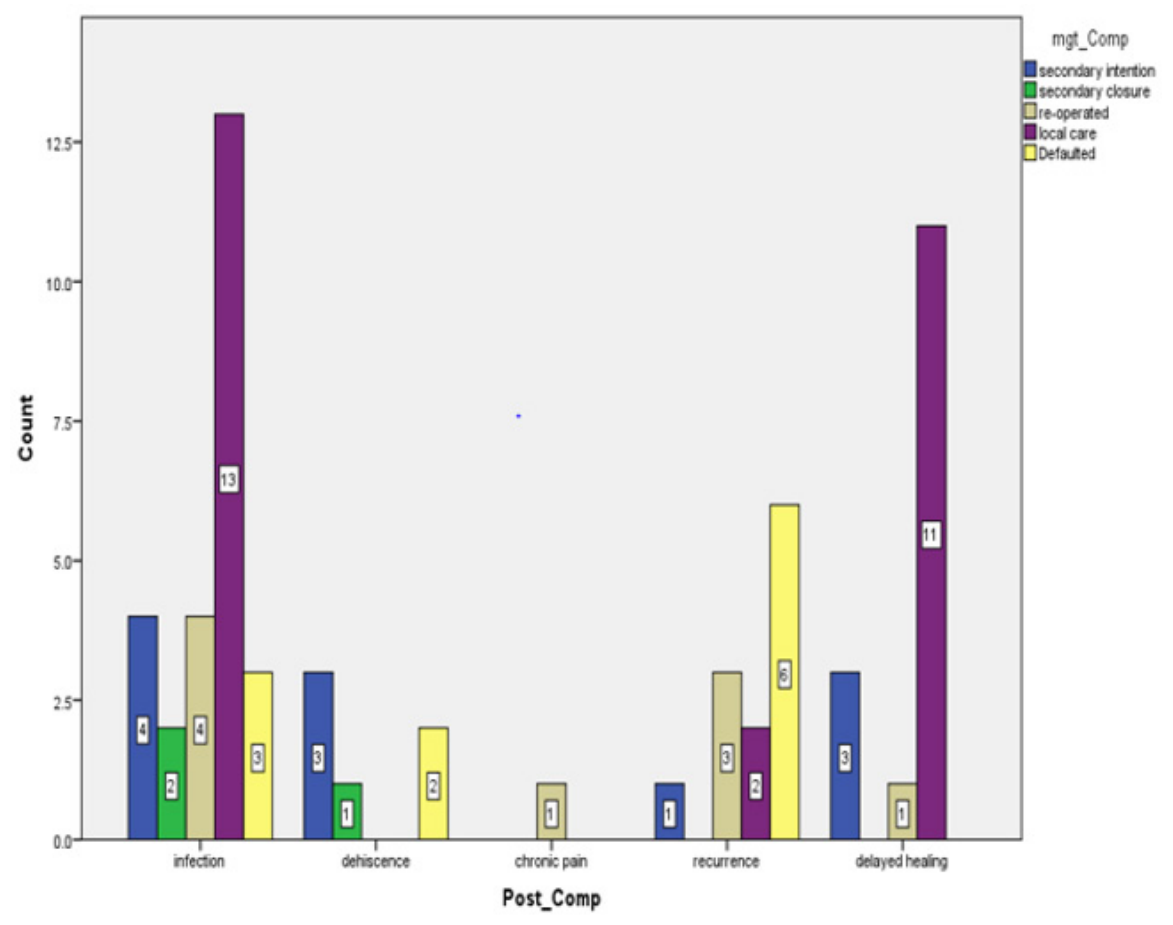

Figure 2: Management of post-surgical complications.

\section{Discussion}

It is well-recognized that this disease negatively affects the quality of life and productivity of young people [10], hence the need for a successful treatment. In view of the wide range of surgical treatment modalities the ideal surgical treatment for pilonidal disease should be simple, requires a minimum hospital stay, with minimum complications and recurrence chances, costeffective, and allows early return to ordinary life and productivity. Minimally invasive excision techniques are hard to compare, as they have been evaluated by different criteria [2] and are not as widely spread as open procedures. Moreover, the minimally invasive techniques have the disadvantage of higher recurrence rate than the excisional methods [2]. Comparative studies of the off-midline techniques are still scarce. However, there is some evidence of excision and off-midline closure superiority $[11,12]$. Although the karydakis procedure is not a new technique; it still remains an important option in the less-privileged settings. It is not technically demanding and reasonably achieves most of these treatment goals [13-15]. Karydakis procedure successfully addresses the three causative factors: the invader (loose hair), the force (deep and narrow natal cleft), and the vulnerability of the raphe $[5,16]$. Other flap techniques; like Limberg flap; may also address the three causative factors but are technically challenging [17]. An additional advantage of the Karydakis procedure it offers a better cosmos than the Limberg flap.
The recurrence rate is the most important variable for the comparative assessment of different modes of treatment, but there is no generally accepted definition of what constitutes a recurrence [2]. In many publications recurrence is not defined at all. In this study we defined recurrence as sinus re-appearance following complete healing of operative site. Our recurrence rate was $4.5 \%$ which is comparable to the rate achieved by Kitchen of 4\% [18]. A short hospital stay is an important factor for favoring any treatment modality. The hospital stay in our series was 5.36 days for the Karydakis group. This clearly exceeds what was reported by Karydakis (3 days) [5] and Kitchen (4days) [18]. One reason for our relatively prolonged hospital stay although the surgical technique had been standardized in our department, we lacked consensus on the timing of wound drain removal. This had reflected on the length of the hospital stay. Another factor which impacted the hospital stay was the in-hospital use of the negative pressure wound therapy (NPWT) for patients who developed wound infection and/ or dehiscence. Furthermore, it is significant to mention that all patients were admitted one or two days prior to surgery and some patient's hospital stay was extend for other medical reasons which may have reflected also on the calculated length of hospital stay (admission - to- discharge).

Wound complications are not rare, particularly when the wound has been closed primarily. Karydakis [5] reported $8.5 \%$ wound-related complications, slightly better than our $10.5 \%$ 
rate. Making sure no active infection at the time of the definitive surgery is a major factor in the prevention of wound-related complications. Antibiotic prophylaxis, proper surgical technique and tissue handling, and timely removal of drain are also important contributing factors to complication-free post-operative course. Doll and his colleagues had observed less recurrence in patients having excision with primary closure sometime after incision and drainage of pilonidal abscess compared to those with self-ruptured abscess and granuloma [19]. In this series there is one recurrence in patients who had incision and drainage of abscess before the standard procedure and most recurrences were in the sinus presentation group (i.e. self-ruptured abscess).

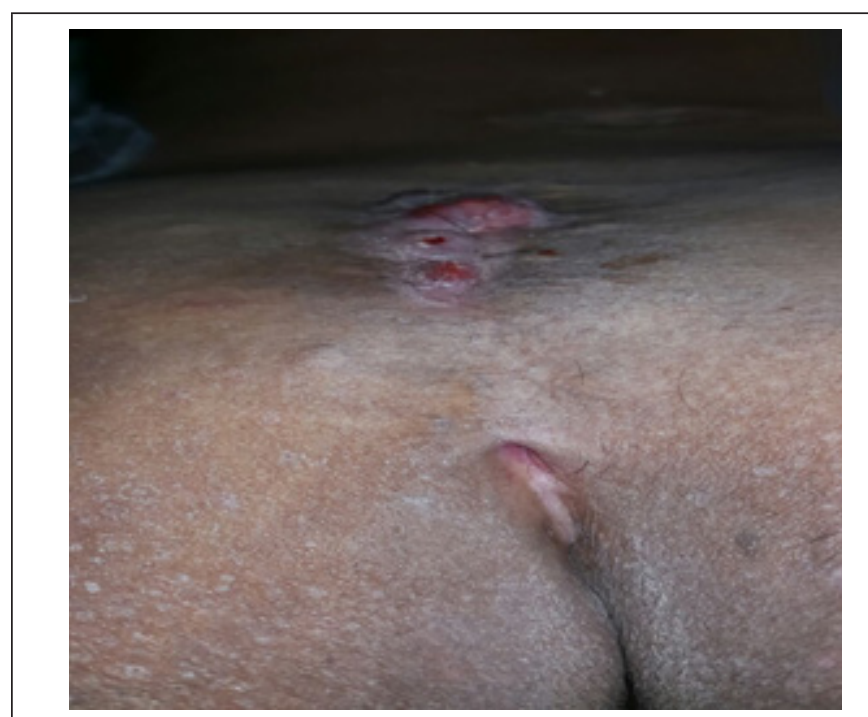

Figure 3: Recurrent pilonidal sinus with off-midline multiple granulomas.

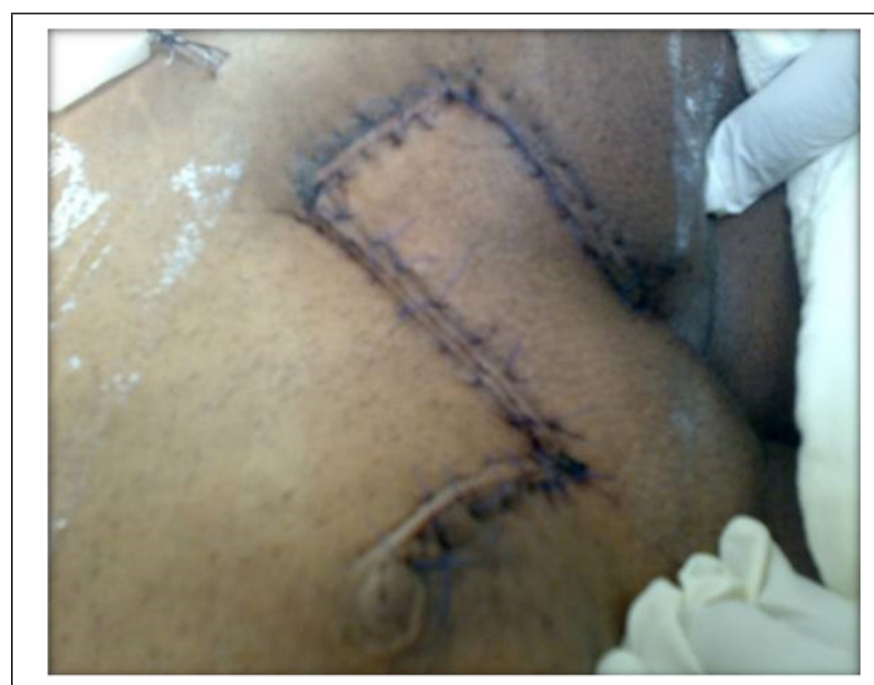

Figure 4: Limberg flap post-operaive view.

Karydakis procedure was also the main option for recurrent cases keeping the Limberg flap as the last resort. The Limberg flap betters Karydakis procedure in terms of recurrence but not in hospital stay and return to work [20]. The main indication for the Limberg flap was recurrence with multiple off-midline sinuses after initial excision and primary closure procedure (Figure 3).
Eight patients had wide excision and closure using Limberg flap technique (Figure 4,5). Although their mean hospital stay was longer than the Karydakis group but still that was less than the open technique group (7.25 Vs 8.7). This is significantly longer than what Jamal and colleagues reported of 4.1 days [10]. The gap is even more when compared to Urhan and colleagues mean hospital stay of 3.7 days [18]. Only one patient developed recurrence after the Limberg flap procedure. Karydakis [5] had followed his patients for 35 years and Keshvari [15] had followed them for 49 months. We had followed up our patients between one week and three and a half years (Mean 11.2 weeks). It was a short follow up duration, but it was relatively close to what Kitchen [18] reported although he had finally concluded that all recurrences had occurred in the first 18 months post-operatively.

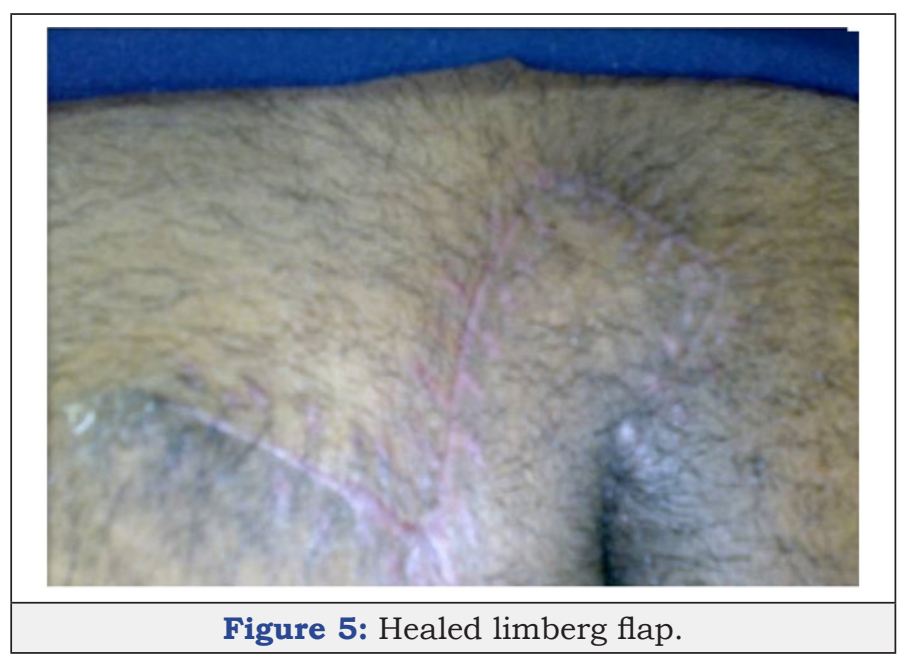

\section{Conclusion}

Pilonidal sinus disease represents a significant proportion of the surgeon's work-load. The Karydakis operation is a relatively simple and effective procedure for cure of the sacrococcygeal pilonidal sinus disease with a low incidence of postoperative complications and recurrence. Modern minimally invasive techniques may surpass the results of this procedure, but they are not widely available for the non-specialized surgeon. Karydakis procedure; although an old technique; remains a successful option for the general surgeon. A pretty novel concept highlighted by this study is that standardization of a procedure among team members is the guarantor of consistent results, not the skills of any individual team member.

\section{Acknowledgment}

The authors would like to acknowledge assistance in data collection of Dr. Hanan Al Kyomi, Dr. Fatema Al Nabhani, Dr. Sara Fatema Zulfiqar Tejani, and Dr. Waad Anas. The authors would like also to acknowledge Dr. Mohamed Salih Mahfouz, PhD, assistance in statistical analysis.

\section{References}

1. Kanat BH, Sözen S (2015) Disease that should be remembered: Sacrococcygeal pilonidal sinus disease and short history. World J Clin Cases 3(10): 876-879. 
2. Igors II, Ommer A (2019) The management of pilonidal sinus. Dtsch Arztebl Int 116(1-2): 12-21.

3. Franklin P, Bendewald, Robert RC (2007) Pilonidal disease. Clinics in Colon and Rectal Surgery 20(2):

4. Bascom J (1980) Pilonidal disease: Origin from follicles of hairs and results of follicle removal as treatment. Surgery 87: 567-572.

5. Karydakis GE (1992) Easy and successful treatment of pilonidal sinus after explanation of its causative process. Aust N Z J Surg 62(5): 385-389.

6. Al Khamis A, McCallum I, King PM, Bruce J (2011) Healing by primary versus secondary intention after surgical treatment for pilonidal sinus. Cochrane Database Syst Rev (4): CD006213.

7. Burnett D, Smith SR, Young CJ (2018) The surgical management of pilonidal disease is uncertain because of high recurrence rates. Cureus 10(5): e2625.

8. Chintapatla S, Safarani N, Kumar S (2003) Sacrococcygeal pilonidal sinus: Historical review, pathological insight and surgical options. Tech Coloproctol 7(1): 3-8.

9. Bascom J, Bascom T (2007) Utility of the cleft lift procedure in refractory pilonidal disease. Am J Surg 193(5): 606-609.

10. Enriquez-Navascues JM, Emparanza JI, Alkorta M, Placer C (2014) Metaanalysis of randomized controlled trials comparing different techniques with primary closure for chronic pilonidal sinus. Tech Coloproctol 18(10): 863-872.

11. Iesalnieks I, Deimel S, Schlitt HJ (2013) Karydakis flap for recurrent pilonidal disease. World J Surg 37(5): 1115-1120.
12. Kitchen PR (1996) Pilonidal sinus: Experience with the Karydakis flap. Br J Surg 83(10): 1452-1455.

13. Karydakis GE (1973) New approach to the problem of pilonidal sinus. Lancet 2(7843): 1414-1415.

14. JGM Smit (2010) The treatment of pilonidal disease. World J Surg 34: 153-157.

15. Keshvari A, Keramati MR, Fazeli MS, Kazemeini A, Meysamie A, et al. (2015) Karydakis flap versus excision-only technique in pilonidal disease. J Surg Res 198: 260-266.

16. Kumar NA, Sutradhar P (2014) Karydakis procedure for sacrococcygeal pilonidal sinus disease: Our experience. Indian J Plast Surg 47(3): 402406.

17. Bali İ, Aziret M, Sözen S (2015) Effectiveness of Limberg and Karydakis flap in recurrent pilonidal sinus disease. Clinics 70(5): 350-355.

18. Jamal A, Shamim M, Hashmi F, Qureshi MI (2009) Open excision with secondary healing versus rhomboid excision with Limberg transposition flap in the management of sacrococcygeal pilonidal disease. J Pak Med Assoc 59(3): 157-160.

19. Doll D, Matevossian E, Hoenemann C, Hoffmann S (2013) Incision and drainage preceding definite surgery achieves lower 20-year long-term recurrence rate in 583 primary pilonidal sinus surgery patients. J Dtsch Dermatol Ges 11: 60-64.

20. Horwood J, Hanratty D, Chandran P, Billings P (2012) Primary closure or rhomboid excision and Limberg flap for the management of primary sacrococcygeal pilonidal disease? A meta-analysis of randomized controlled trials. Colorectal Dis 14(2): 143-151. 